\title{
Seronegative autoimmune hepatitis in children: a single-center experience
}

\author{
A. Islek ${ }^{1}$, H. Keskin ${ }^{2}$ \\ (1) Atatürk University School of Medicine, Department of Pediatric Gastroenterology, 25240, Erzurum, Turkey ; (2) Atatürk University School of Medicine, Department of \\ Pediatrics, 25240, Erzurum, Turkey.
}

\begin{abstract}
Background : Seronegative autoimmune hepatitis (AIH) is a diagnostic challenge with unclear prognosis. This study describes the features and outcomes of seronegative AIH in children.

Patients and methods : Patients under 18 years of age, who had been diagnosed with AIH between April 2014 and April 2020, were retrospectively evaluated. Seronegative AIH was identified by the absence of the three conventional non-organ-specific autoantibodies (anti-nuclear antibody [ANA], anti-smooth muscle antibody [ASMA], and anti-liver kidney microsomal [anti-LKM] type 1 antibody), alongside the characteristic AIH liver histopathology and a positive response to immunosuppressive therapy in the absence of other liver diseases.

Results : The study included 54 patients with AIH. $15(27.77 \%)$ were seronegative at the time of diagnosis. 13 of the 15 seronegative patients presented with acute hepatitis or acute liver failure (ALF). Mean follow-up duration was $\mathbf{2 7 . 4 8}$ months in seronegative patients. Two seronegative patients had lymphocytopenia on admission, and, although the liver disease improved after corticosteroid treatment, they developed aplastic anemia (AA). Other seronegative patients responded well to immunosuppressive therapy.

Conclusions : Patients with seronegative AIH present frequently with acute hepatitis or ALF. AIH diagnosis can be confirmed by observing the effects of corticosteroid therapy in seronegative patients with characteristic AIH liver histopathological features. However, the presence of lymphocytopenia in seronegative patients is a sign of bone marrow failure. (Acta gastroenterol. belg., 2021, 84, 305-310).
\end{abstract}

Key words : Seronegative autoimmune hepatitis, lymphocytopenia, aplastic anemia, diagnosis, prognosis.

\section{Introduction}

Autoimmune hepatitis (AIH) diagnosis is based on the presence of non-organ-specific antibodies (anti-nuclear antibody [ANA], anti-smooth muscle antibody [ASMA], and anti-liver kidney microsomal [anti-LKM] type 1 antibody), hypergammaglobulinemia, and characteristic liver histopathological features (1). Criteria from the International Autoimmune Hepatitis Group (IAIHG) (2) or the European Society for Pediatric Gastroenterology Hepatology and Nutrition (ESPGHAN) (3) are used to diagnose $\mathrm{AIH}$ in children. However, AIH diagnosis can be difficult because, even with AIH present, these criteria may not be met (4). In recent years, a new term seronegative AIH - has been introduced to describe AIH diagnoses that do not present conventional autoantibody positivity but fulfil the other AIH criteria (4). It has been suggested that seronegative AIH accounts for less than $5 \%$ of all AIH cases $(3,4)$. Due to its rarity, little information is available in children (5). However, an association between seronegative $\mathrm{AIH}$ and hematological disorders - such as aplastic anemia (AA), which has a poor prognosis - has been reported in a few studies among populations of children $(5,6)$. Early diagnosis and treatment prevent progressive liver disease. While AIH responds well to immunosuppressive therapy (3), the treatment and prognosis for seronegative AIH have not been clearly described for children $(5,6)$. This study, therefore, sought to fill this gap by discussing the features and prognosis of seronegative AIH in patients under 18 years of age.

\section{Patients and methods}

The medical records of patients under 18 years of age, who had been diagnosed with AIH between April 2014 and April 2020 in the tertiary hospital pediatric gastroenterology unit, were retrospectively analyzed. Seropositive AIH was diagnosed by the presence of at least one conventional non-organspecific antibody (ANA, ASMA, or anti-LKM type 1), hypergammaglobulinemia, and specific histopathologic features with the exclusion of other liver diseases in patients with elevated aminotransferases. Patients with alanine transaminase (ALT) and aspartate aminotransferase levels above the normal limit for over one month, in whom other liver diseases were excluded, underwent liver biopsy for AIH. Interface hepatitis, portal lymphocyte and plasma cell infiltration, rosette formation, and emperipolesis were considered consistent with AIH. Hypergammaglobulinemia was defined as immunoglobulin $\mathrm{G}$ ( $\mathrm{IgG}$ ) levels exceeding the reference ranges for age (7). Liver diseases other than $\mathrm{AIH}$ were excluded (Table 1). In addition, lysosomal acid lipase deficiency was excluded in seronegative cases. Lysosomal acid lipase activity above $0.37 \mathrm{nmol} / \mathrm{punch} / \mathrm{h}$ was considered normal. ANA, ASMA, and anti-LKM type 1 levels were examined by indirect immunofluorescence. Levels above the $1 / 40$ titer were considered positive, as mentioned in a study by the IAIHG (2). Patients who tested positive for ANA and ASMA were defined as having type $1 \mathrm{AIH}$, and those who tested positive for

Correspondence to: Ali Islek, MD, Associate Professor of Pediatric Gastroenterology, Atatürk University School of Medicine, Department of Pediatric Gastroenterology, 25240, Erzurum, Turkey. Phone : +90 5057664380. Email : islekali@hotmail.com

Submission date : 17/06/2020 Acceptance date : 16/10/2020 
Table 1. — Excluded liver diseases

\begin{tabular}{|l|l|}
\hline Liver diseases & Tests \\
\hline $\begin{array}{l}\text { Drug-induced liver } \\
\text { disease }\end{array}$ & History and liver biopsy \\
\hline $\begin{array}{l}\text { Acute or chronic viral } \\
\text { hepatitis }\end{array}$ & $\begin{array}{l}\text { Hepatitis A, B, C, E, EBV, and parvovirus } \\
\text { B19 serology }\end{array}$ \\
\hline $\begin{array}{l}\text { Alpha-1 antitrypsin } \\
\text { deficiency }\end{array}$ & Serum alpha-1 antitrypsin \\
\hline Wilson disease & $\begin{array}{l}\text { Ceruloplasmin, 24-hour urine copper test, } \\
\text { Kayser-Fleischer rings on ophthalmolo- } \\
\text { gical examination }\end{array}$ \\
\hline Hemochromatosis & Transferrin, iron, and ferritin \\
\hline $\begin{array}{l}\text { Pediatric metabolic } \\
\text { diseases }\end{array}$ & $\begin{array}{l}\text { Tandem mass spectrometry, plasma amino } \\
\text { acids, and urine organic acid tests }\end{array}$ \\
\hline
\end{tabular}

EBV : Epstein-Barr virus.

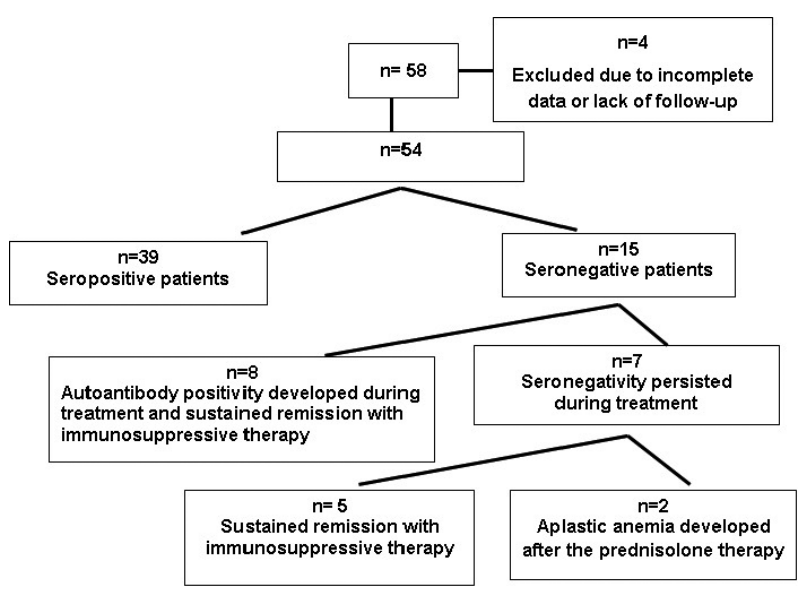

Figure 1. - Flow chart of patients with autoimmune hepatitis

anti-LKM type 1 were defined as having type $2 \mathrm{AIH}$ $(1,2)$. Seronegative AIH was identified by the absence of ANA, ASMA, and anti-LKM type 1, the present of characteristic AIH liver histopathology, and a positive response to immunosuppressive therapy in the absence of other liver diseases (5). Scores were calculated in line with the 2008 Simplified Criteria for the Diagnosis of Autoimmune Hepatitis (2). Magnetic resonance cholangiography (MRCP) was performed on all patients to determine whether they should be diagnosed with autoimmune sclerosing cholangitis (ASC). Intrahepatic or extrahepatic multiple strictures and dilatation on MRCP led to an ASC diagnosis. Esophagogastroduodenoscopy was performed in patients with clinical and laboratory findings of cirrhosis and/or portal hypertension.

Remission was defined as aminotransferase levels within normal ranges, IgG normalization, and negative autoantibody tests (3).

Prednisolone, at a dose of $2 \mathrm{mg} / \mathrm{kg} / \mathrm{d}$ (maximum 60 $\mathrm{mg} / \mathrm{d}$ ), was administered to all patients diagnosed with $\mathrm{AIH}$. The dose was reduced to $5 \mathrm{mg} / \mathrm{d}$ in six to eight weeks, correlating with the decrease in aminotransferases. In the event of relapse, or if aminotransferases did not continue to decrease, azathioprine treatment was initiated. In the event of no response to prednisolone or azathioprine therapy, mycophenolate mofetil (MMF) was used as a second-line therapy. If remission did not occur, MMF was substituted with cyclosporine A or tacrolimus. Ursodeoxycholic acid (UDCA) was used in ASC cases.

\section{Statistical Analysis}

Statistical analyses were performed using the Statistical Package for the Social Sciences (SPSS) software, version 24 (SPPS, Chicago, USA). The results were expressed as mean \pm standard deviation (SD). Since not all parameters were normally distributed, the Chisquared, Mann-Whitney U and Kruskal-Wallis $\mathrm{H}$ tests were employed to compare groups. $\mathrm{P}$ values $<0.05$ were considered statistically significant.

\section{Results}

\section{Patients}

58 patients with AIH were followed up during the dates specified above. Four were excluded due to incomplete data and lack of follow-up. Thus, 54 patients were included. In 39 (72.22\%), at least one test for the conventional autoantibodies of ANA, ASMA or anti-LKM was positive (seropositive patients) (Figure 1). Mean ages for type $1 \mathrm{AIH}$, type $2 \mathrm{AIH}$, and seronegative AIH were 11.02, 5.13, and 5.69 years, respectively $(\mathrm{p}<0.01)$. ALT levels did not significantly differ between type $1 \mathrm{AIH}$, type $2 \mathrm{AIH}$, and seronegative $\mathrm{AIH}$ (i.e., $656.12 \pm 102.45 \mathrm{u} / \mathrm{L}, 702.32 \pm 129.11 \mathrm{u} / \mathrm{L}$, and $699 \pm 201.65 \mathrm{u} / \mathrm{L}$, respectively). Lymphocyte counts also did not significantly differ between type $1 \mathrm{AIH}$, type 2 AIH, and seronegative AIH $(2805 \pm 354 \mu 1,2912 \pm 478 \mu$ l, and $3150 \pm 446 \mu$ l, respectively) (Table 2 ).

\section{Presentations}

12 patients $(40.00 \%)$ with type $1 \mathrm{AIH}$, one patient (11.11\%) with type $2 \mathrm{AIH}$, and two patients (13.33\%) with seronegative AIH presented with cirrhosis and/or portal hypertension stigmas (hematemesis, splenomegaly, and hypersplenism). Eight patients with type $1 \mathrm{AIH}$ (26.67\%), eight patients with type 2 AIH $(88.89 \%)$, and 13 patients with seronegative AIH (86.67\%) presented with acute hepatitis or acute liver failure (ALF) (nausea, vomiting, fatigue, abdominal pain, jaundice, dark-colored urine, and acholic stools). Six patients with type $1 \mathrm{AIH}$ $(20.00 \%)$ presented with asymptomatic transaminase elevation. Four patients with type 1 AIH (13.33\%) presented with insidious onset (progressive fatigue, amenorrhea, headache, and/or weight loss) (Table 2).

\section{Seronegative patients}

Tests for all three conventional antibodies were negative in 15 patients. Seropositivity developed during follow-up in eight of these (three only ANA, one ANA with ASMA and four anti-LKM), while seronegativity persisted in the other seven (Figure 1). The median time to development of seropositivity was 27 days (18-51 days). 13 of the 15 seronegative patients presented with acute 
Table 2. - Patient characteristics

\begin{tabular}{|c|c|c|c|c|}
\hline Types of AIH & $\begin{array}{l}\text { Type } 1 \\
\mathrm{n}: \mathbf{3 0}\end{array}$ & $\begin{array}{c}\text { Type } 2 \\
\text { n : } 9\end{array}$ & $\begin{array}{l}\text { Seronegative } \\
\mathrm{n}: 15\end{array}$ & $\mathbf{p}$ \\
\hline Gender (F/M) & $18 / 12$ & $5 / 4$ & $9 / 6$ & $<0.01$ \\
\hline \multicolumn{5}{|l|}{ Age of diagnosis (years) } \\
\hline $\begin{array}{l}\text { Mean } \\
\text { Median (range) }\end{array}$ & $\begin{array}{c}11.02 \\
12.40(6.30-17.24)\end{array}$ & $\begin{array}{c}5.13 \\
6.25(3.72-8.50) \\
\end{array}$ & $\begin{array}{c}5.19 \\
7.50(5-12.12) \\
\end{array}$ & $<0.01$ \\
\hline \multicolumn{5}{|l|}{ Presentation symptoms [n (\%)] } \\
\hline Complications of cirrhosis and $\mathrm{PH}$ & $12(40.00)$ & $1(11.11)$ & $2(13.33)$ & $<0.01$ \\
\hline Acute hepatitis or ALF & $8(26.67)$ & $8(88.89)$ & $13(86.67)$ & $<0.01$ \\
\hline Asymptomatic transaminase elevation & $6(20.00)$ & $0(0.00)$ & $0(0.00)$ & $<0.01$ \\
\hline Insidious onset & $4(13.33)$ & $0(0.00)$ & $0(0.00)$ & $<0.01$ \\
\hline \multicolumn{5}{|l|}{ Follow-up period (months) } \\
\hline Mean (range) & $\begin{array}{l}29.26 \\
(11-62)\end{array}$ & $\begin{array}{c}26.65 \\
(12-59)\end{array}$ & $\begin{array}{l}27.48 \\
(4-58)\end{array}$ & 0.11 \\
\hline \multicolumn{5}{|l|}{ Autoantibodies (n) } \\
\hline ANA & 30 & 0 & 0 & \multirow{3}{*}{-} \\
\hline ASMA & 11 & 0 & 0 & \\
\hline Anti-LKM & 0 & 9 & 0 & \\
\hline \multicolumn{5}{|l|}{ Laboratory } \\
\hline $\operatorname{ALT}(\mathrm{u} / \mathrm{L})(\mathrm{mean} \pm \mathrm{SD})$ & $656.12 \pm 102.45$ & $702.32 \pm 129.11$ & $699.10 \pm 201.65$ & 0.22 \\
\hline $\mathrm{IgG} x$ upper limit (mean $\pm \mathrm{SD})$ & $1.44 \pm 0.53$ & $1.32 \pm 0.41$ & $1.04 \pm 0.34$ & $<0.01$ \\
\hline Lymphocyte count $(\mu \mathrm{l})($ mean $\pm \mathrm{SD})$ & $2805 \pm 354$ & $2912 \pm 478$ & $3150 \pm 446$ & 0.43 \\
\hline
\end{tabular}

ALF : acute liver failure ; ALT : alanine aminotransferase ; ANA : anti-nuclear antibody ; anti-LKM : anti-liver kidney microsomal type 1 ; ASMA : anti-smooth muscle antibody ; IgG : immunoglobulin G ; AIH : autoimmune hepatitis ; PH : portal hypertension ; SD: standard deviation.

hepatitis (seven) or ALF (six). The remaining two patients presented with cirrhosis and portal hypertension stigmas. Two of the 15 seronegative patients who subsequently underwent hematopoietic stem cell transplantation (HSCT) had lymphocytopenia on admission.

\section{IgG levels}

IgG levels were lower in seronegative AIH than in type 1 and type 2 AIH $(1.04 \pm 0.34 \mathrm{x}$ the upper limit of normal, $1.44 \pm 0.53 x$ the upper limit of normal, and $1.32 \pm 0.41 x$ the upper limit of normal, $\mathrm{p}<0.01$, respectively). IgG levels did not significantly differ between type 1 and type $2 \mathrm{AIH}$ $(1.44 \pm 0.53 \mathrm{x}$ the upper limit of normal and $1.32 \pm 0.41 \mathrm{x}$ the upper limit of normal, $\mathrm{p}=0.11$, respectively) (Table 2). IgG levels were within normal ranges at the time of diagnosis in eight of the 15 seronegative patients. However, these IgG levels decreased by an average of $30.70 \%$ after treatment in these eight patients.

\section{Liver biopsy and histopathology}

Liver biopsy was performed in all patients. Portal lymphocyte, plasma, or eosinophil infiltration and interface hepatitis were observed in all patients, while emperipolesis was observed in $15(27.77 \%)$. Cirrhosis histopathology was observed in 17 (31.48\%) cases, and varying degrees of portal-to-portal or portal-to-central fibrosis were detected in all other cases. Histological ASC (including bile duct damage, acute and/or chronic cholangitis, or biliary periportal hepatitis) were observed in three cases $(5.55 \%)$.

\section{Comorbid autoimmune diseases}

A total of ten comorbid autoimmune events were observed in eight patients with type 1 AIH : ASC in five cases, ulcerative colitis in two ASC cases, Hashimoto's thyroiditis in two cases, and celiac disease in one case. AIH was diagnosed in celiac patients whose aminotransferases did not normalize, despite a six-month gluten-free diet.

\section{Treatment}

Treatment began with only prednisolone in all cases, with additional UDCA in ASC cases. Azathioprine was added to treatment at various times in 45 (83.33\%) patients. Second- and third-line immunosuppressive drugs (MMF, tacrolimus, or cyclosporine A) were used in four $(7.40 \%)$ cases in which remission was not achieved or maintained.

\section{Follow-up duration and remission}

Follow-up durations were not significantly different between type $1 \mathrm{AIH}$, type $2 \mathrm{AIH}$, and seronegative AIH (29.26 months, 28.65 months, and 27.48 months, $\mathrm{p}=0.11$, respectively). Two seronegative patients treated with only prednisolone developed AA. Five seronegative patients, whose autoantibody negativity persisted, remained in remission at a mean time of 24 months with prednisolone and azathioprine therapy. Second- or thirdline treatments were used in a few seropositive patients to maintain remission (mentioned above). 
Table 3. - Laboratory results of cases with AA

\begin{tabular}{|l|c|c|c|c|}
\hline & \multicolumn{2}{|c|}{ Patient 1 } & \multicolumn{2}{c|}{ Patient 2 } \\
\hline Age $($ years $)$ & \multicolumn{2}{|c|}{$\mathbf{5 . 1 0}$} & Initial & 2nd week \\
\hline & Initial & 2nd week & 1300 & 232 \\
\hline ALT $(\mathrm{u} / \mathrm{L})$ & 636 & 106 & 1100 & 185 \\
\hline AST $(\mathrm{u} / \mathrm{L})$ & 726 & 110 & 118 & 46 \\
\hline GGT $(\mathrm{u} / \mathrm{L})$ & 41 & 52 & 8.10 & 3.10 \\
\hline Total bilirubin $(\mathrm{mg} / \mathrm{dL})$ & 18.40 & 5.90 & 6.60 & 1.60 \\
\hline Direct bilirubin $(\mathrm{mg} / \mathrm{dL})$ & 10.80 & 2.80 & 1.20 & 1.20 \\
\hline INR & 1.80 & 1.30 & 9.20 & 8.10 \\
\hline IgG $(\mathrm{g} / \mathrm{L})$ & 7.10 & 6.40 & 13.60 & 11.30 \\
\hline Hemoglobin $(\mathrm{g} / \mathrm{dL})$ & 10.20 & 9.20 & 6800 & 1100 \\
\hline WBC $(\mu \mathrm{l})$ & 10400 & 3200 & 1730 & 340 \\
\hline Lymphocyte count $(\mu \mathrm{l})$ & 530 & 240 & 258000 & 82000 \\
\hline Platelet $(\mu \mathrm{l})$ & 356000 & 93000 & & \\
\hline
\end{tabular}

ALT : alanine aminotransferase ; AST : aspartate aminotransferase ; GGT : gamma glutamyl transferase ; IgG : immunoglobuline G ; INR : international normalized ratio ; WBC : white blood cell count.

\section{Seronegative cases complicated with AA}

Progressive bone marrow failure developed in two seronegative patients, who presented with acute cholestatic hepatitis. IgG levels were within the normal range in these patients. Portal lymphoplasmacytic inflammation, interface hepatitis, and rosette formation, compatible with AIH, were observed in their liver biopsies. Stage 3-4 fibrosis, according to the Ishak scoring system (8), was also observed in the liver biopsies. Lymphocytopenia was present in both patients on admission. Hypertransaminasemia and lymphocytopenia lasted for two months before corticosteroid treatment. Despite improvement in their liver tests after corticosteroid treatment, pancytopenia became evident after the second week (Table 3). HSCTs from the patients' siblings were performed at the fourth and eighth months after diagnosis, respectively, because of the development of bone marrow failure. No lymphocytopenia was observed in any of the other seronegative patients.

\section{Liver transplantation (LT)}

Three patients with cirrhosis underwent LT (at the $24^{\text {th }}, 32^{\text {nd }}$ and $36^{\text {th }}$ months of follow-up, respectively). LT indications were recurrent variceal bleeding in two patients and growth retardation with refractory pruritis in a patient with ASC.

\section{Scores for AIH}

Scores based on the 2008 simplified AIH classification system were 8 in 11 cases, 7 in 32, 6 in six, 5 in three, and 4 in two (2).

\section{Discussion}

Although the prevalence of pediatric AIH has increased in recent years, diagnostic difficulties are sometimes (not uncommon) still ongoing (9). The presence of autoantibodies is important for diagnosing AIH (3). Knowledge about seronegative cases in children is limited $(4,6)$. Reported adult experiences suggest that it is similar to classic $\mathrm{AIH}$, except for autoantibody positivity, but there is no consensus for the diagnosis of seronegative $\mathrm{AIH}$ (5). It has been suggested that autoantibodies may be negative in fewer than $5 \%$ of all AIH cases $(3,4,6)$. In this study, autoantibodies were negative in 15 patients $(27.77 \%)$ on admission. In contrast to adults, children with AIH have lower autoantibody titers (3). In the European Society for Pediatric Gastroenterology Hepatology and Nutrition (ESPGHAN) position paper, positivity at a dilution $\geq 1: 20$ for ANA and ASMA and $\geq$ $1: 10$ for anti-LKM was considered clinically significant (3). The threshold titers for ANA, ASMA and anti-LKM in our laboratory are 1/40. Tests at lower dilutions are not routinely performed and in many laboratories this is the case. Having a higher rate of seronegativity than reported in the literature may be due to the high thresholds. A negative autoantibody result alone should not exclude the diagnosis of AIH (10). Majority of seronegative cases (13 of 15) presented with acute cholestatic hepatitis or ALF. Although autoantibody frequency in cases with acute hepatic presentations, including ALF, is uncertain, it has been suggested that autoantibodies may be positive at low titers or negative in most of these cases (11-14). It has been hypothesized that conventional autoantibodies may be delayed in their appearance due to the early, intense antigen-antibody complex, which disrupts the assay (13). Seropositivity subsequently developed in more than half of the patients with acute presentation in this study, supporting the suggested high early seronegativity rates in patients with acute disease manifestation.

Although AA associated with fulminant hepatic failure (FHF) and hepatitis is well known (15), there are concerns about an association between seronegative AIH and hematological disorders (4,6,16-18). In one study (15) patients who underwent LT after FHF and developed AA had lower white blood counts, lymphocyte counts, and platelet counts compared to patients with FHF who 
underwent LT but did not develop AA. An undetermined acute viral infection has been held responsible for the development of AA and FHF in patients who have undergone LT (19). In the present research, interface hepatitis and advanced fibrosis were established in the two seronegative patients who developed AA. Therefore, the authors surmise that hepatitis, in these patients who consequently developed AA, was a chronic process rather than part of an acute viral event. Bone marrow failure developed in the two seronegative patients in this study who presented with acute cholestatic hepatitis, and lymphocytopenia was present in them at the time of diagnosis. These patients exhibited the characteristic AIH liver histology and had a score of 4 based on the 2008 Simplified Criteria for the Diagnosis of AIH. Liver tests in these two cases improved with prednisolone therapy alone, and they underwent HSCT. These findings are distinctive features different from those present in FHF-associated AA.

Adult studies indicate that seronegative $\mathrm{AIH}$ is a variant of AIH and that its management should be equivalent to that for seropositive AIH (5). In this study seronegative patients were treated in the same way as seropositive patients. Because seronegative and seropositive patient follow-up durations were similar, the authors can say that treatment response was also similar-except in the two patients who developed AA. Therefore, the authors suggest that the prognosis of seronegative $\mathrm{AIH}$ is similar to that of seropositive AIH. Maggiore et al. (4) have reported poor prognosis in seronegative AIH with lymphocytopenia. Their research, which is the only patient series study of seronegative AIH in the literature, states that, of 13 children with lymphocytopenia, disease was resolved in only one after immunosuppressive therapy, while 12 presented a blood disorder (4).

It has been suggested that hypergammaglobulinemia may not be present in up to one in four cases of type $1 \mathrm{AIH}$, type $2 \mathrm{AIH}$, and seronegative $\mathrm{AIH}(5,6,20)$. IgG levels were within the normal range for age in half of the seronegative patients $(53.33 \%)$ in the present study. Two patients, who developed AA, also had normal gammaglobulin levels. However, IgG levels decreased by an average of $30.70 \%$ after corticosteroid treatment in seronegative patients. IgG levels decreased by $23 \%$ and $27 \%$ after corticosteroid treatment in patients who developed AA. Although, decrease in IgG levels after immunosuppressive treatment in patients with $\mathrm{AIH}$ an expected finding, it has no prognostic value for seronegative AIH. The authors recommend careful evaluation in terms of the presence of lymphocytopenia at diagnosis or follow-up in seronegative cases, especially in the absence of hypergammaglobulinemia. However, they also recommend evaluating corticosteroid response to avoid delayed treatment of AIH in seronegative cases if liver histopathology is compatible with AIH. The presence of portal plasma cell infiltration with interface hepatitis is an important finding for the diagnosis of AIH. If there is difficulty in identifying plasma cells, immunostaining for multiple myeloma oncogene 1 (MUM1) may be useful (21).

The study has limitations. Since it was a retrospective study, the importance of antibodies such as anti-soluble liver antigen (anti-SLA) and anti-liver cytosol type 1 (anti-LC-1) could not be evaluated in seronegative cases. Seronegative AIH prevalence could not be calculated according to low antibody thresholds due to the reason stated above.

\section{Conclusion}

Patients with seronegative AIH present frequently with acute hepatitis or ALF. Although seronegativity with normal globulin levels makes the diagnosis of AIH difficult, if other causes of liver disease are ruled out and specific histopathological findings of AIH are determined in liver biopsy, observing corticosteroid efficacy seems to be a better way of aiding exact diagnosis and avoiding delayed treatment. Delayed seropositivity within the first two months of treatment is not uncommon. However, the presence of lymphocytopenia in seronegative patients is a predictor of future bone marrow failure and should be follow-up carefully.

\section{Author contributions}

AI substantially contributed to the conception and design of the study. $\mathrm{AI}$ and $\mathrm{HK}$ contributed to the acquisition, analysis and interpretation of the data. AI drafted the article and made critical revisions related to the intellectual content of the manuscript and approved the final version of the article to be published.

\section{Institutional review board statement}

The study was reviewed and approved by the Committee of Ethics at the Faculty of Medicine, Atatürk University, Erzurum, Turkey (B.30.2.ATA.0.01.00/110)

\section{Conflict-of-interest statement}

The authors declared that no conflict of interest exists.

\section{Funding sources}

No funds received.

\section{References}

1. MIELI-VERGANI G., VERGANI D. Paediatric autoimmune liver disease. Arch. Dis. Child., 2013, $98: 1012-1017$.

2. HENNES EM., ZENIYA M., CZAJA AJ., PARÉS A., DALEKOS GN., KRAWITT EL., et al. Simplified criteria for the diagnosis of autoimmune hepatitis. Hepatology., 2008, $48: 169-176$.

3. MIELI-VERGANI G., VERGANI D., BAUMANN U., CZUBKOWSKI P., DEBRAY D., DEZZOFI A., et al. Diagnosis and Management of Pediatric Autoimmune Liver Disease : ESPGHAN Hepatology Committee Position Statement. J. Pediatr. Gastroenterol. Nutr., 2018, 66 : 345-360.

4. CZAJAA.Autoantibody-negative autoimmunehepatitis. Dig Dis Sci., 2012,57: 610-624. 
5. MAGGIORE G., SOCIE G., SCIVERES M., ROQUE-AFONSO AM., NASTASIO S., JOHANET C., et al. Seronegative autoimmune hepatitis in children : Spectrum of disorders. Dig. Liver Dis. 2016, 48 : 785-791.

6. CUELLO LL., ARNAL IR., ROMERO RG., ARGARATE CH. Seronegative Autoimmune Hepatitis : Description of Two Paediatric Cases. An. Pediatr. (Barc)., 2018, 88: 285-286.

7. LO SF. Reference intervals for laboratory tests and procedures. In KLIEGMAN RM., STANTON BF., ST GEME III JW., SCHOR NF., eds. Nelson Textbook of Pediatrics 20 $0^{\text {th }}$ ed. Philadelphia : Elsevier Inc; 2016 : 3464-3473.

8. ISHAK K., BAPTISTA A., BIANCHI L., CALLEA F., DE GROOTE J., GUDAT F., et al. Histological grading and staging of chronic hepatitis. $J$. Hepatol., 1995, 22 : 696-699.

9. MACK CL., ADAMS D., ASSIS DN., KERKAR N., MANNS MP., MAYO MJ., et al. Diagnosis and management of autoimmune hepatitis in adults and children : 2019 practice guidance and guidelines from the American Association for the study of liver diseases. Hepatology., 2019, Dec 21. doi : 10.1002/hep.31065

10. PATHAK S., KAMAT D. Autoimmune Hepatitis in Children. Pediatr. Ann., 2018, 47 : e81-e86.

11. VERGANI D., ALVAREZ F., BIANCHI FB., CANCADO., MACKAY LR., MANNS MP., et al. Liver autoimmune serology : a consensus statement from the committee for autoimmune serology of the International Autoimmune Hepatitis Group. J. Hepatol., 2004, 41 : 677-683.

12. NARKEWICZ MR., HORSLEN S., BELLE SH., RUDNICK DA., NG VL., ROSENTHAL P., et al. Prevalence and Significance of Autoantibodies in Children with Acute Liver Failure. J. Pediatr. Gastroenterol. Nutr., 2017, 64 : 210-17.

13. MCFARLANE IG. Pathogenesis of autoimmune hepatitis. Biomed Pharmacother., 1999, $53: 255-263$.
14. GREGORIO GV., MCFARLANE B., BRACKEN P., VERGANI D., I G., et al. Organ and non-organ specific autoantibody titres and IgG levels as markers of disease activity : a longitudinal study in childhood autoimmune liver disease. Autoimmunity., 2002, 35 : 515-519.

15. MOLINA RA., KATZIR L., RHEE C., INGRAM-DRAKE L., MOORE T., KROGSTAD P., et al. Early evidence of bone marrow dysfunction inchildren with indeterminate fulminant hepatic failure who ultimately develop aplastic anemia. American Journal of Transplantation., 2004, 4 : 1656-1661.

16. IKEDA T., MORIMOTO A., NAKAMURA S., YOKOYAMA K., HAYASE T., OH Y., et al. A Marked Decrease in CD4-positive Lymphocytes at the Onset of Hepatitis in a Patient With Hepatitis-associated Aplastic Anemia. $J$. Pediatr. Hematol. Oncol., 2012, 34 : 375-377.

17. SAVAGE WJ., DERUSSO PA., RESAR LM., CHEN AR., HIGMAN MA., LOEB DM., et al. Treatment of hepatitis-associated aplastic anemia with high-dose cyclophosphamide. Pediatr. Blood Cancer., 2007, 49 : 947 951.

18. RASMUSSEN LK, STENBØG EV, KERNDRUP GB, HASLE H. Autoimmune Hepatitis and Seronegative Hepatitis Associated With Myelodysplastic Syndrome in Children. J. Pediatr. Hematol. Oncol., 2016, 38 : e274-e277.

19. SAFADI R., OR R, ILAN Y., NAPARSTEK E., NAGLER A., KLEIN A., et $a l$. Lack of known hepatitis virus in hepatitis-associated aplastic anemia and outcome after bone marrow transplantation. Bone Marrow Transplant., 2001, 27 : 183-190.

20. MIELI-VERGANI G., HELLER S., JARA P., VERGANI D., CHANG MH., FUJISAWA T., et al. Autoimmune hepatitis. J. Pediatr. Gastroenterol. Nutr., 2009, 49 : 158-164.

21. TRILLING AU., RAMOISIAUX L., KOMUTA M., LANTHIER N. Autoimmune hepatitis in a patient with thymectomy : the benefit of histology and MUM-1 immunostaining. Acta Gastroenterol. Belg., 2019, 82 : 550-551. 